\title{
Finite element simulation of fixed dental prostheses made from PMMA -Part I: Experimental investigation under quasi-static loading and chewing velocities
}

\author{
Alexander SCHMIDT'1, Ihssane KIDIDANE², Maximiliane Amelie SCHLENZ , Bernd WÖSTMANN¹, Stefan KOLLING² \\ and Peer SCHRADER ${ }^{2}$ \\ ${ }^{1}$ Department of Prosthodontics, Dental Clinic, Justus-Liebig-University, Schlangenzahl 14, 35392 Giessen, Germany \\ ${ }^{2}$ Institute of Mechanics and Materials, Technische Hochschule Mittelhessen, Wiesenstrasse 14, 35390 Giessen, Germany \\ Corresponding author, Alexander SCHMIDT; E-mail: alexander.schmidt@dentist.med.uni-giessen.de
}

\begin{abstract}
Material properties are of high clinical relevance, even though in vitro laboratory setups may differ from clinical conditions. Therefore, the aim of the present study was to investigate the fracture behavior of three-unit bridge restoration (Telio CAD) with different test velocities (1.0 mm/min International Organization for Standardization (ISO) standard speed/ $130 \mathrm{~mm} / \mathrm{s}$ mean chewing velocity) and to provide crucial validation experiments for the upcoming Part 2 of our study, in which FEA on such temporary restorations will be conducted. Local strains were detected using digital image correlation (DIC). The material exhibited significantly different responses at different test velocities, and the forces at fracture were found to be much smaller at chewing velocity $(130 \mathrm{~mm} / \mathrm{s})$ than in the quasistatic test. Overall, the results of the present study show that characteristics pertaining to material behavior can change significantly with increasing chewing velocity, and that fracture forces decrease with increasing test velocity.
\end{abstract}

Keywords: Finite element analysis, Materials testing, Dental materials, PMMA, Digital image correlation

\section{INTRODUCTION}

Material properties and fracture behavior are of high clinical relevance, given that dental biomaterials have to resist the high chewing velocities and masticatory forces produced by the patient. Therefore, these materials are typically investigated via in vitro laboratory tests before they are tested via in vivo test setups on patients. Investigations on the materials' behavior and properties are commonly performed using classical experiments, such as three-point bending tests, tensile tests, or compression tests ${ }^{1,2)}$.

One of the challenges with dental biomaterials is that they are usually tested via in vitro investigations in the laboratory, followed by in vivo test setups on patients. This is a time-consuming and cost-intensive procedure, because numerous test series are necessary.

As an example, for provisional materials, International Organization for Standardization (ISO) 10477: Polymer-based crown and veneering materials ${ }^{2)}$ is usually used as the standard, with a specified testing velocity of $1.0 \mathrm{~mm} / \mathrm{min}\left(0.0166^{-} \mathrm{mm} / \mathrm{s}\right)$ exemplifying quasi-static-loading. However, given that different test velocities lead to differing measurements in material properties for polymeric materials ${ }^{3)}$, using a test velocity of only $1.0 \mathrm{~mm} / \mathrm{min}$ fails to consider the differences in subsequent material behavior at different chewing velocities. Moreover, a review of the literature has revealed the mean chewing velocity to be $130 \mathrm{~mm} / \mathrm{s}$ $(7,800 \mathrm{~mm} / \mathrm{min})^{4-8)}$.

Furthermore, laboratory testing poses an additional problem, given that laboratory conditions may differ

Color figures can be viewed in the online issue, which is available at J-STAGE.

Received Jun 15, 2020: Accepted Aug 19, 2020

doi:10.4012/dmj.2020-230 JOI JST.JSTAGE/dmj/2020-230 from clinical conditions (e.g., in terms of environment, saliva, loading rate, and temperatures). Moreover, it is not always technically feasible to integrate all clinically relevant conditions directly into an in vitro test setup to mimic all clinical conditions. Therefore, all chosen parameters, such as test velocities, carry a risk of failure within clinical application. Thus, with regard to the mechanical parameters of dental materials, we must conclude that this area of research has not been sufficiently investigated in current literature ${ }^{9)}$, especially when the strong dependence of material behavior on loading rate is considered ${ }^{3)}$, and understanding of this aspect of dental biomaterial science and its clinical implications is sparse. For example, if bending or fracture tests are conducted with a test velocity of $1.0 \mathrm{~mm} / \mathrm{min}$, which is much lower than the estimated loading rate in patients, the results may be significantly different only because of the different loading rates. However, if a material clinically differs significantly from its expected behavior, as expected from in vitro testing, there is a decisively increased risk for reduced life span of the restoration. When this restoration is later applied to patients, much higher chewing velocities can lead to fracture or even complete loss of the restoration ${ }^{10,11}$. This leads to an increased risk of inhalation or swallowing of the restoration; pain and/or caries are also possible consequences. As a result, a de novo visit to the dentist would be necessary, resulting in further expenditure of time and money.

Furthermore, dental biomaterials are typically classified under medical devices and not under pharmaceuticals, which gives many manufacturers the opportunity to introduce a new product to the dental market without having to conduct a clinical trial on 
patients according to medical device regulations, provided that data from comparable materials are already available. This is possible if a given material can be assigned to a material group that has already been tested. Nevertheless, materials of the same material class may differ significantly in terms of their material characteristics.

Therefore, it would be very helpful to establish an equivalent approach in dental biomaterials, similar to the 3R principle (replace, reduce, refine) by Russel and Burch ${ }^{12)}$, which is used in animal experiments. A similar procedure would also be desirable for investigations on patients. Next to the classical in vitro laboratory tests, the use of finite element analysis (FEA) offers such an opportunity. FEA allows a numerical simulation based on previously determined material properties of a biomaterial under consideration. This analysis has already been used successfully for many years in technical areas, for example, in mechanical engineering ${ }^{13)}$.

Given that there are few studies on dental biomaterials that focused on the effects of chewing speed, we have developed a practical model of a three-unit fixed dental prosthesis (FDP) using the typical chewing velocity and, for comparison, the test speed according to ISO standards. The aim of the present study is to investigate the fracture behavior of three-unit bridge restoration (Telio CAD) with different test velocities (1.0 $\mathrm{mm} / \mathrm{min}$ ISO standard speed/ $130 \mathrm{~mm} / \mathrm{s}$ mean chewing velocity) and to provide crucial validation experiments for the upcoming Part 2 of our study, in which FEA on such temporary restorations will be conducted. Local strains and macroscopic fracture processes were detected using digital image correlation (DIC).

\section{MATERIALS AND METHODS}

For the model, we selected a bridge restoration in the left upper jaw, which was simulated in the present study using FEA and a temporary dental biomaterial (Telio CAD). To test different properties at different chewing speeds, the investigations were conducted once at 1.0 $\mathrm{mm} / \mathrm{min}$ and at an average chewing speed of $130 \mathrm{~mm} / \mathrm{s}$. Here, we were able to detect critical strains locally with the help of DIC. Furthermore, we were able to calculate the fracture probabilities of the prostheses under certain loads. Both the local strains and the distribution of fracture forces are particularly relevant for clinically close examinations, especially for common chewing speeds, extreme loads such as hard food (e.g., cherry stone), or for patients with bruxism, given that these are often excluded from clinical studies.

\section{Materials and specimen manufacturing}

For specimen mounting, an idealized chamfer preparation (preparation taper $6^{\circ}$; stump height $6 \mathrm{~mm}$; anatomical circular and occlusal substance removal 1 $\mathrm{mm}$ ) was created using computer-aided design (CAD) (millhouse, Hofheim-Wallau, Germany) for teeth FDI 25 and 27 with missing tooth 26 , as patient equivalent. Based on the CAD files, a bridge mounting was produced
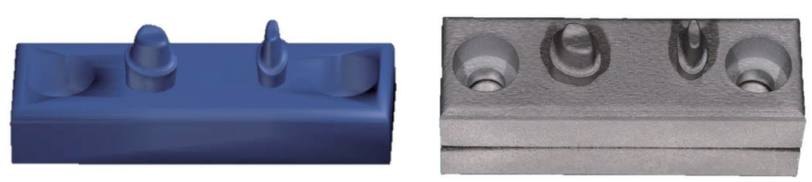

Fig. 1 CAD data for specimen mounting (left) and final specimen mounting via laser sintering (right).
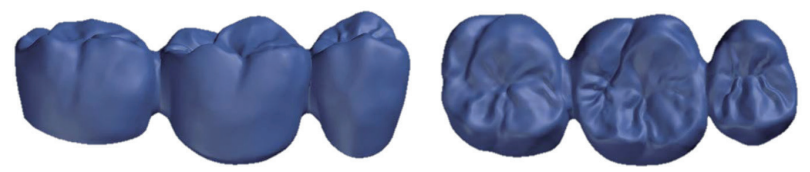

Fig. 2 CAD data for bridge specimen from vestibular (left) and occlusal (right).

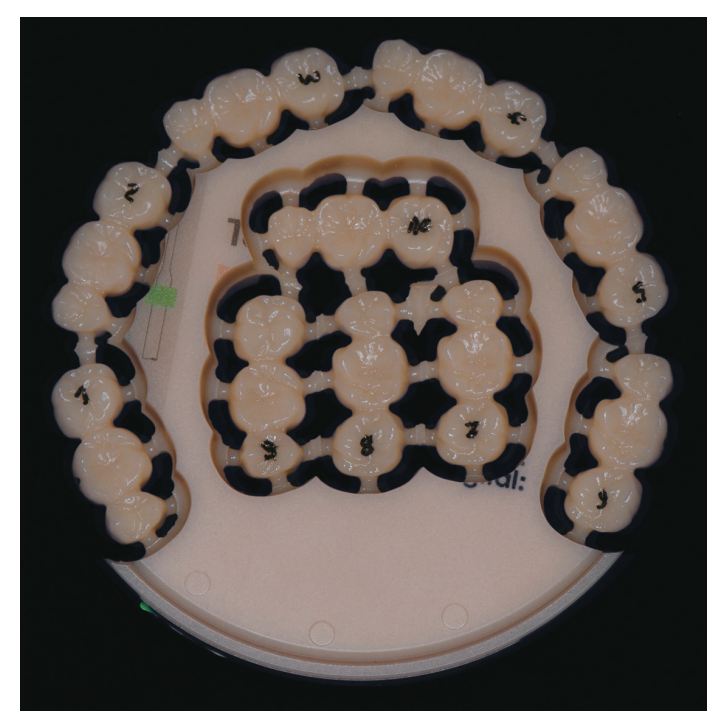

Fig. 3 Milled bridges in Telio CAD blank (lower part was used for fixing in milling machine, numbered for later identification).

via computer-aided manufacturing (CAM) using laser sintering (Fig. 1).

Based on the dies of teeth 25 and 27 (FDI), a threeunit FDP bridge was first designed using CAD (Fig. 2).

The bridges were milled from Telio CAD-LT discs (shade A3, diameter $98.5 \mathrm{~mm}$, thickness $20.0 \mathrm{~mm}$; crosslinked polymethyl methacrylate (PMMA); percentage weight $99.5 \%$, pigments $<1 \%$, no further fillers ${ }^{14-16)}$; Ivoclar Vivadent, Schaan, Lichtenstein) in a milling center (millhouse) using a 5-axis milling machine (Mikron HSM 400U LP, GF Machining Solutions, Schorndorf, Germany). Four bridges were milled from the interior, and six from the exterior per circular blank, and then numbered (Fig. 3).

\section{Antagonist position (spherical)}

To create a unique and repeatable condition of the antagonist of the bridge, a chrome sphere (grade class 


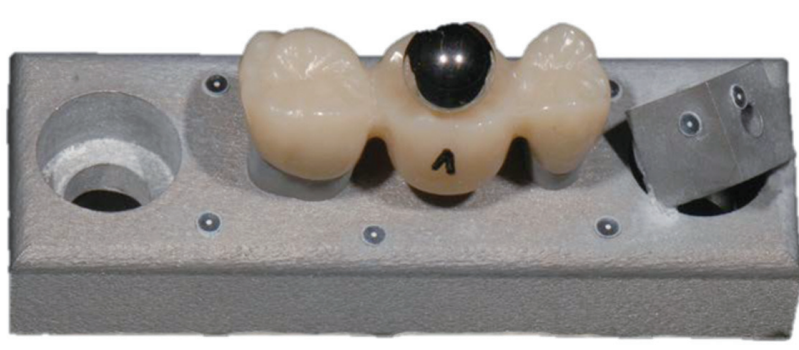

Fig. 4 Bridge specimen with antagonist (sphere) on specimen mounted with reference cube.

5 according to DIN 5401:2002-08), $7 \mathrm{~mm}$ in diameter, was used as antagonist. For digitizing, a high-definition laboratory scanner (ATOS gom, GOM, Braunschweig, Germany; accuracy $<4 \mu \mathrm{m}$ ) was used. This enabled the determination of the center coordinate of the sphere in the later finite element simulation and allowed for the prediction of the reproducibility of positioning.

For this purpose, the support of the bridge construction was aligned horizontally with reference points in the laboratory scanner. A steel cube $(10 \times 10 \times 10$ $\mathrm{mm}$ ) served as reference and was attached to the specimen holder, such that three surfaces could be scanned equally during the scanning process. The sphere was then positioned in the central fissure of the bridge pontic (Fig. 4).

To reduce possible reflections, the bridge and the sphere were powdered with a titanium-dioxide powder (High-Resolution Scanning Spray Powder, 3M, St. Paul, MN, USA). A total of 40 scans were performed: 20 after the sphere was repositioned, and 20 after the sphere was left in place. Each of these 40 scans consisted of ten scans, which were overlaid to form a point cloud. Afterward, the point clouds were polygonised and exported to an STL format.

The data were imported into measurement software (gom Inspect 2018, GOM). A coordinate system was then created using the reference cube. Afterward, the center of the sphere was constructed.

Thus, it was possible to determine the deviations between the centers of the repositioned spheres within the different scans. From these ten distances, the mean value was calculated.

\section{Experimental setup}

For the component tests at different chewing velocities, the bridges were luted on the patient equivalent using a glass ionomer cement (Ketac Cem Aplicap, 3M). The bridge, which was sprayed with a stochastic color pattern beforehand, was placed on the specimen holder, and the cementum was left to cure for an hour. The antagonist was placed on the bridge specimen and pressed down on the bridge using a compression stamp, achieving threepoint contact with the chewing surface of the middle tooth.

The bridges were tested at a haul-off speed of 1.0 $\mathrm{mm} / \mathrm{min}$, which is commonly used in the testing of dental



Fig. 5 Test setup for low-velocity examination $(1.0 \mathrm{~mm} /$ min) using ARAMIS system.

prostheses, and at a higher velocity of $130 \mathrm{~mm} / \mathrm{s}$ to estimate the mandible movements and typical velocities during mastication.

Low-velocity tests were performed on a servo electric universal testing machine, Inspekt 5 (Hegewald \& Peschke, Nossen, Germany) with a $5 \mathrm{kN}$ load cell. During the tests' runtime, compression stamp, and specimens were continuously filmed using an ARAMIS 3D Motion and Deformation Sensor (GOM) with a $12 \mathrm{MP}$ image resolution to determine the machine path locally without the influence of machine stiffness and to gain local strain measurements, which were later evaluated using GOM Correlate (GOM) software. For the DIC measurements, a measuring distance of $285 \mathrm{~mm}$ and a stereo angle of $22^{\circ}$ were selected. Furthermore, camera lenses with a $50 \mathrm{~mm}$ focal length were used. For the measurements, an image acquisition rate of $4 \mathrm{~Hz}$ was chosen. According to the manufacturer of the given system, the accuracy of position measurement is $\pm 20 \mu \mathrm{m}$ for a measurement volume of $1 \times 1 \times 1 \mathrm{~m}$. The test setup is shown in Fig. 5. Overall, 13 tests were performed with this setup at a velocity of $1.0 \mathrm{~mm} / \mathrm{min}$.

The tests at chewing velocity were conducted using an MTS Bionix servohydraulic test system (MTS Systems, Eden Prairie, MN, USA) with a $15 \mathrm{kN}$ load cell. The specimens were filmed using a MotionXtra N4 highspeed camera (Imaging Solutions, Eningen, Germany) with 1 megapixels resolution to measure the machine path locally. An image acquisition rate of $18.95 \mathrm{kHz}$ was selected for this purpose. Furthermore, a camera lens with a focal length of $105 \mathrm{~mm}$ was used. For a better overview, the test setup is shown in Fig. 6. With this setup, 9 valid experiments were performed.

\section{Statistical procedure}

All statistical analyses were performed using MATLAB 2017b (MathWorks, Natick, MA, USA). The fracture forces during the component tests were investigated using the two-parameter Weibull distribution $(2 \mathrm{PW})^{17)}$

$$
P(F)=1-\exp \left[-\left(\frac{F}{\eta}\right)^{\beta}\right]
$$




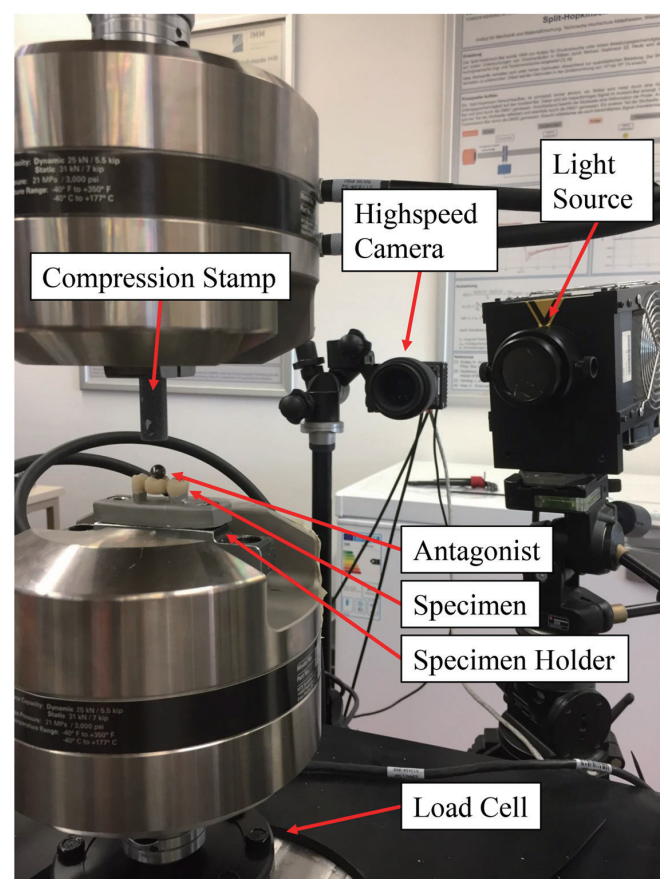

Fig. 6 Test setup for high-speed examination $(130 \mathrm{~mm} / \mathrm{s})$ using high-speed camera.

with the probability $P$ of the occurrence of the force value $F$, scale parameter $\eta$, and shape parameter $\beta$. Here, $\eta$ is the force value at which $1-\exp (-1)=63.2 \%$ of the specimens are expected to fail during the experimental procedure, whereas $\beta$ is a measure for the dispersion of forces at fracture. The parameters $\eta$ and $\beta$ were determined via residual sum of squares. The Weibull probability estimator $p_{i}=i /(N+1)$ was used to assign an equally distributed probability to the measured forces at fracture. Here, $i$ is the position of the measured fracture force in the ascendingly ordered sample of $N$ test results.

\section{RESULTS}

\section{Antagonist position (spherical)}

The deviation of the center of the sphere after repositioning resulted in a mean deviation of 6.4 $\mu \mathrm{m}( \pm 0.67 \mu \mathrm{m})$. The deviation of the sphere without repositioning resulted in a mean deviation of $1.9 \mu \mathrm{m}$ $( \pm 0.45 \mu \mathrm{m})$.

For better illustration, the sphere position in Fig. 7 was superimposed according to the best possible alignment of the smallest distances to each other (bestfit algorithm $)^{18)}$.

\section{Force-displacement curves and DIC}

The results of the tests performed at velocities of 1.0 $\mathrm{mm} / \mathrm{min}$ and $130 \mathrm{~mm} / \mathrm{s}$ are visualized in Fig. 8. For the low-velocity tests, the measured force-displacement curves are observed to correlate strongly with each other. The measured curves at a loading velocity of 130

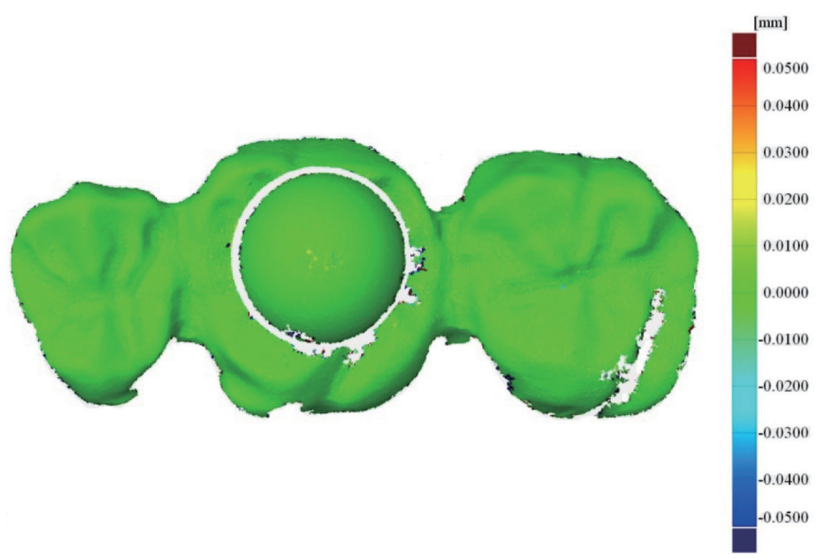

Fig. 7 Sphere position after superimposition (deviations from master model are displayed in color).

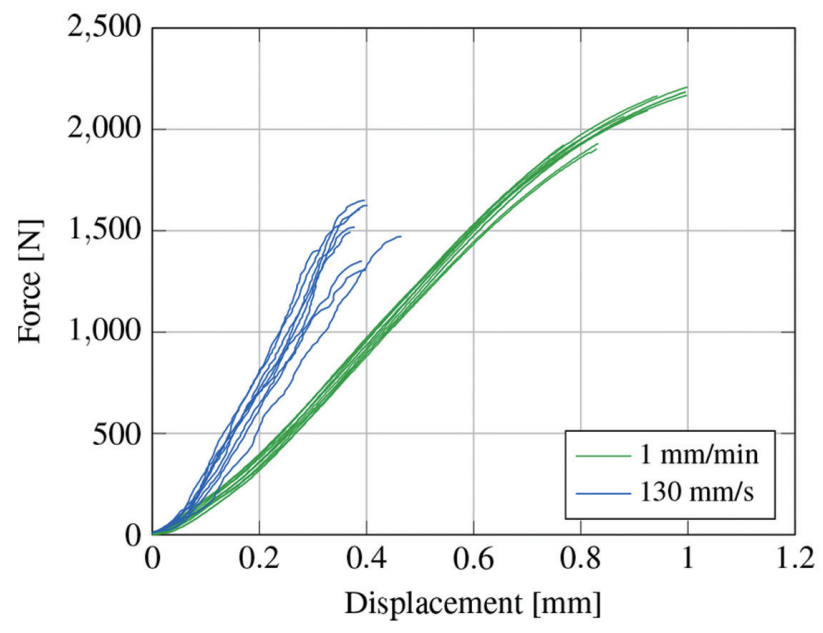

Fig. 8 Results for test velocities of $1.0 \mathrm{~mm} / \mathrm{min}$ and 130 $\mathrm{mm} / \mathrm{s}$.

$\mathrm{mm} / \mathrm{s}$ show a slightly higher dispersion, and a smaller force at fracture.

During the low-velocity tests, the specimens could be observed to be slightly bent out of the specimen holder, as shown in Fig. 9. This was further indicated by the fact that the destroyed specimens could be removed from the specimen holder by hand, although these specimens were previously adhesively bonded to the holder with the glass ionomer cement. For the tests at chewing velocity, this movement could not be observed. Here, leverage had to be used to remove the specimens from the specimen holder, indicating that the adhesive bond did not fail.

For the low-velocity tests, after the local determination of the position of the compression stamp, the strain on the surface of the specimen was determined using DIC. Information on the local strains obtained using DIC is visualized in Fig. 10. Here, the last DIC image before fracture is shown with the corresponding (unitless) maximum principal strain, which was determined using the Correlate software. It 
should also be noted that the facet size in the DIC was approximately $0.4 \mathrm{~mm}$.

\section{Distribution of fracture forces}

For both setups, a significant scattering of the fracture forces can be observed, which was investigated using 2PW distribution. The distribution of the fracture forces and their corresponding parameters are shown in Fig. 11 and Table 1 , respectively. The mean forces at fracture were $2,045.6 \pm 121.6 \mathrm{~N}$ at $1.0 \mathrm{~mm} / \mathrm{min}$ and $1,491.2 \pm 114.7$ $\mathrm{N}$ at $130 \mathrm{~mm} / \mathrm{s}$.

\section{Fracture behavior}

Distinct characteristic fracture patterns could also be observed for both test velocities. During the low-velocity

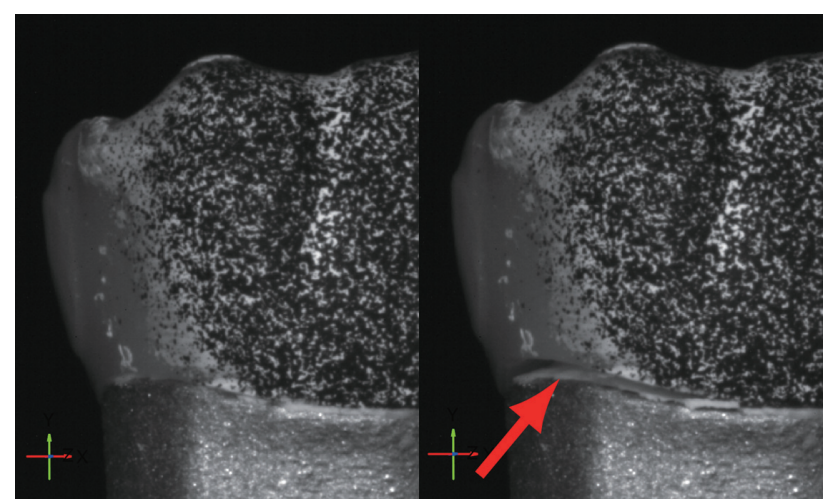

Fig. 9 Example of initial situation (left) and removal of test specimen during experimental investigation (right).

The red arrow shows the bending of the sample from the sample holder.

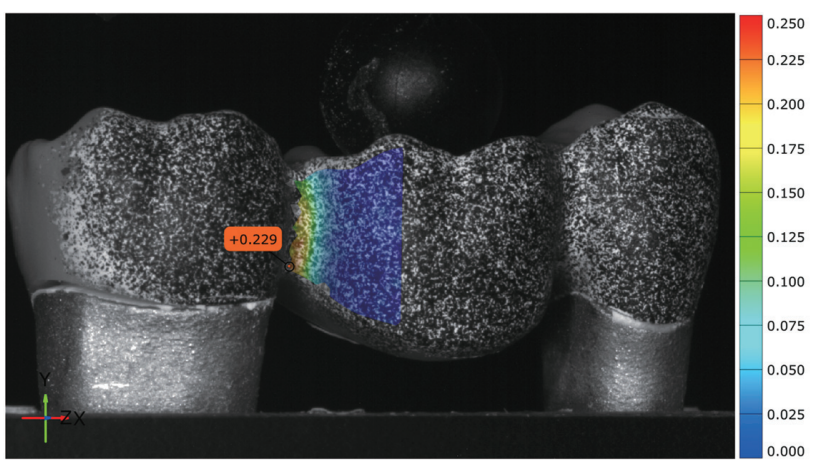

Fig. 10 Results for maximum principal strains (unitless) determined using DIC. tests (Fig. 12a), cracks mostly started to propagate from the left pontic. Afterward, in the middle of the FDP, the cracks branched to both the direction of the antagonist and the direction of the right pontic. Within the conducted tests, separation of the fractured parts occurred only in the fewest cases, meaning that the structural integrity of the component was usually still reliable, even after

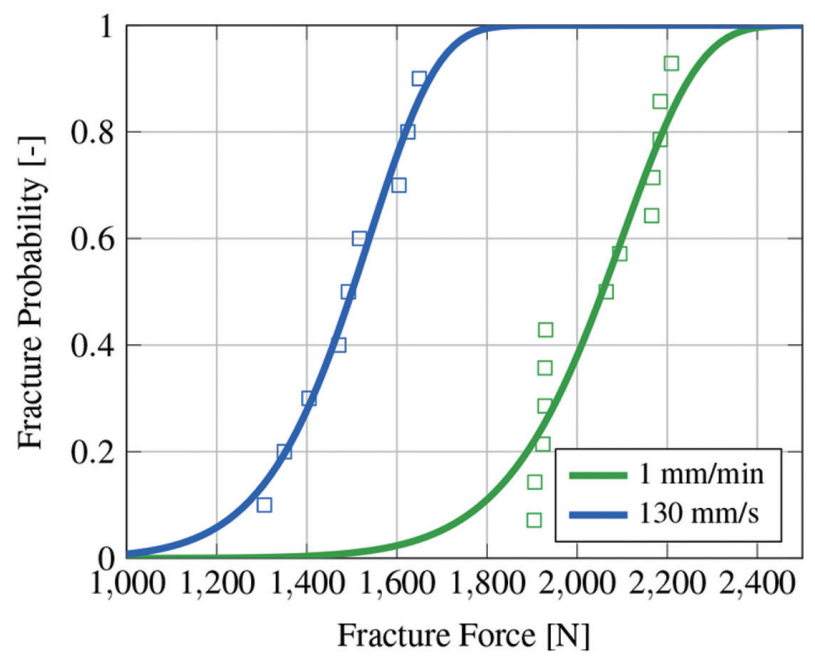

Fig. 11 Distribution of fracture forces for different test velocities.

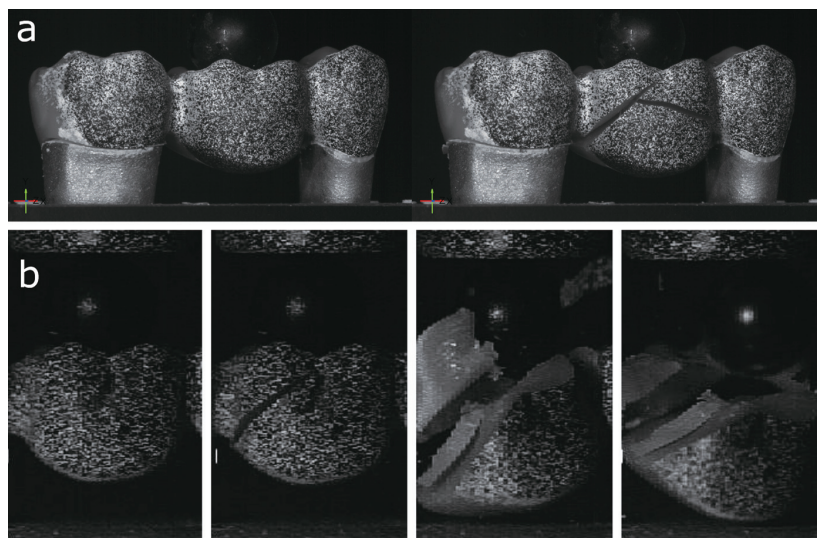

Fig. 12 Fracture behavior during low velocity testing (1.0 $\mathrm{mm} / \mathrm{min}$ ) at the beginning of loading (left) after the end of loading (complete fracture, right) (a) and during high velocity testing $(130 \mathrm{~mm} / \mathrm{s})$ at the beginning of loading (left) and during loading until complete fracture (from left to right) (b).

Table 1 Fracture forces and calculated 2PW distribution parameters

\begin{tabular}{cccc}
\hline Test velocity & Scale parameter $\eta[\mathrm{N}]$ & Shape parameter $\beta[-]$ & Residual Sum of Squares $[-]$ \\
\hline $1.0 \mathrm{~mm} / \mathrm{min}$ & $2,113.39$ & 13.39 & 0.0972 \\
$130 \mathrm{~mm} / \mathrm{s}$ & $1,550.66$ & 11.00 & 0.0122 \\
\hline
\end{tabular}


failure. At high velocities, on the other hand, the middle tooth was destroyed completely in most cases, with a small connector remaining in the rear part of the prosthesis. This can be observed in Fig. 12b. Here, the crack was also observed to start in the lower side of the left pontic. However, owing to the impact energy, the middle tooth was destroyed completely.

\section{DISCUSSION}

We decided to create a model based on the use of PMMA to test homogeneous initial situations within the material. This method can then be applied to more complex materials, such as hybrid ceramics. Furthermore, given that temporary treatments fulfil a wide range of purposes within the prosthodontic dentistry ${ }^{19,20)}$, a typical temporary material (Telio CAD) available on the dental market was selected for the present study. This material is a cross-linked PMMA (percentage weight $99.5 \%$, pigments $<1 \%$ ) with no further fillers ${ }^{14-16}$. It can be milled in dental practice in a chairside (directly on the patient) milling machine via the dental CAD/CAM workflow, using intraoral scanners and in-house milling machines. Through the use of an industrial blank, in combination with production in an external milling center, high standardization could be achieved for the investigations in the present study $^{9,21-24)}$. A previous study has also shown that the highest accuracy could be achieved using a 5 -axis milling machine ${ }^{25}$. Meanwhile, given the risk that heterogeneous materials could strongly influence the properties of material behavior, a homogeneous material was chosen for the present research. More importantly, Telio CAD has already been investigated in previous studies and has proven itself clinically $^{24,26,27)}$.

A bridge specimen in the posterior region was chosen because, in that region, the highest masticatory loads, compared to those in the anterior region, can occur ${ }^{28,29)}$. An ideal chamfer preparation was selected, because this allowed a uniform and homogeneous structure for the bridge construction ${ }^{30)}$. Otherwise, slice preparation at the point of proximal contact could have led to incorrect behavior within the CAD construction. Meanwhile, a typical luting cement was used to allow close clinical examination. Higher fracture loads have also been found in a previous investigation when the specimen was luted ${ }^{311}$. A steel sphere, on the other hand, was used as antagonist, given that this type of antagonist has already been tested in previous studies and is therefore representative of a typical procedure. To ensure a reproducible position for the antagonist, a chromiumsteel sphere with a diameter of $7 \mathrm{~mm}$ was used to ensure high dimensional stability ${ }^{32-35}$. To enable clinically close examination, the sphere used was rolled into a position that was checked in prior to the experiment. This positioning made it possible to perform a similar clinical examination, given that, physiologically, an antagonist contact is normally a multi-point contact.

The test velocities were chosen in accordance with the ISO 10477 standard $^{2)}$. In a previous study by Alt et al., a higher speed of $200 \mathrm{~mm} / \mathrm{min}$ was used to investigate fracture strength measurements in bridge constructions ${ }^{9}$. Although chewing speed varies from person to person, for the patient-equivalent situation in the present research, a mean speed of $130 \mathrm{~mm} / \mathrm{s}$ was chosen based on previous studies ${ }^{4-8)}$. It should be emphasized that the observed oscillations of the measurement curves at 130 $\mathrm{mm} / \mathrm{s}$ are caused by elastic waves that are introduced by the impact of the compression stamp. However, the amplitude of these oscillations is rather small so that the signal does not have to be filtered.

It is also important to discuss why rigid mounting was used for the bridges. In an in vivo setup, natural teeth are characterized by a high degree of inherent mobility. By contrast, the intrinsic mobility of implants is in the range of $10-15 \mu \mathrm{m}$ and, therefore, approximately ten times lower than that of natural teeth ${ }^{36}$. For this reason, the present investigation provides an insight into how the behavior of the patient can be represented. Furthermore, the gap between the restoration and bridge restoration clearly shows how much influence an additional movement of the abutment teeth can have on the occurrence of gaps. Meanwhile, the extent to which the self-natural mobility of the periodontal gap can counteract possible load or fracture of the material remains unclear. This is a clear limitation of the present study.

In the experimental study, displacement measurement using DIC was of high importance. It should be emphasized that using DIC, the machine path can be determined locally. If the machine path was taken directly from the testing machine, the compliance of the machine could possibly have a large influence on the results, especially at a dynamic loading velocity of $130 \mathrm{~mm} / \mathrm{s}$. Although it is possible to accompany the compliance of the testing machine with mathematical calculation, local displacement measurement using DIC provides more accurate results.

Next to testing machine compliance, possible deformation/measuring inaccuracy of the specimen holder and the influence of the adhesive bonding used between holder and specimen must also be considered. As can be seen in Fig. 8, the initial gradients of the force-displacement curves in both the quasi-static and high-speed tests are very similar, indicating that settling processes were present, which are independent of the loading rate. Because preliminary investigations ${ }^{3)}$ have revealed that the initial elastic modulus of the tested PMMA is highly rate-dependent, this behavior must not be considered part of the behavior of the material. Especially in later FEA, the observed compliance of the specimen holder will inevitably lead to some deviations between simulation and experimental results if the specimen holder and adhesive joint are not considered in the simulation.

Different types of consequences of strains in everyday clinical practice must also be distinguished. On one hand, the stress on the material leads to bending and thus to formation of a gap (Fig. 9). This gap formation can either be the result of decementation due to loss of adhesive 
bond between the restoration and cement, or the stress itself can lead to decementation. On the other hand, cracks may occur in the material, which may result in complete loss of the restoration ${ }^{37)}$. There are numerous subtypes of cracks in different dental biomaterials. In principle, any type of crack or fracture in the material is undesirable in everyday clinical practice. However, a complete fracture of the temporary restoration or an unphysiological behavior of the material, which can be recognized directly by the patient, is considered to be better, because a large gap in the stump teeth that has existed for a long period of time can often lead to the occurrence of initial carious lesions on the tooth stump, up to the complete destruction of the tooth. Nonetheless, based on the results of the present study, both decementation with an existing temporary restoration and complete fracture of the temporary restoration can be assumed to possibly occur. In both cases, a new dental treatment would be necessary.

\section{CONCLUSION}

It should be noted that the measured forces at fracture are much larger than the chewing forces expected during mastication ${ }^{38-40}$. This permits the conclusion that the bridge used in these experiments should be able to withstand normal chewing loads without further complications, even at chewing velocity.

Overall, the results of the present study show that the characteristics of the material can change significantly with increasing chewing velocity and that the forces at which fracture can occur in the material can decrease with increasing test velocity. This is of great significance for future studies, given that investigations should not be limited to low test speeds. Furthermore, DIC makes it possible to investigate the behavior not only after a fracture is observed but also during the fracture process itself, allowing more accurate statements to be made about the fracture behavior.

\section{ACKNOWLEDGMENTS}

We acknowledge financial support from the Flexi Funds of the Research Campus of Central Hessen. We gratefully acknowledge the support of Kay Frendel during the production of the test samples.

\section{REFERENCES}

1) Ferry JD. Some reflections on the early development of polymer dynamics: Viscoelasticity, dielectric dispersion, and self-diffusion. Macromolecules 1991; 24: 5237-5245.

2) International Organization for Standardization. ISO 10477:2018, Dentistry: Polymer-based crown and veneering materials 2018.

3) Schmidt A, Schrader P, Frendel K, Schlenz MA, Wöstmann B, Kolling S. Is the assumption of linear elasticity within prosthodontics valid for polymers? - An exemplary study of possible problems. Dent Mater J 2021; 40: 52-60.

4) Bates JF, Stafford GD, Harrison A. Masticatory function - a review of the literature: (II) Speed of movement of the mandible, rate of chewing and forces developed in chewing. J
Oral Rehabil 1975; 2: 349-361.

5) Gawriolek K, Gawriolek M, Komosa M, Piotrowski PR, Azer SS. Kinematic modeling of normal voluntary mandibular opening and closing velocity -initial study. J Prosthodont 2015; 24: 279-286.

6) Komino M, Shiga H. Changes in mandibular movement during chewing of different hardness foods. Odontology 2017; 105: 418-425.

7) Lepley C, Throckmorton G, Parker S, Buschang PH. Masticatory performance and chewing cycle kinematics-are they related? Angle Orthod 2010; 80: 295-301.

8) Uesugi H, Shiga H. Relationship between masticatory performance using a gummy jelly and masticatory movement. J Prosthodont Res 2017; 61: 419-425.

9) Alt V, Hannig M, Wöstmann B, Balkenhol M. Fracture strength of temporary fixed partial dentures: CAD/CAM versus directly fabricated restorations. Dent Mater 2011; 27: 339-347.

10) Penate L, Basilio J, Roig M, Mercade M. Comparative study of interim materials for direct fixed dental prostheses and their fabrication with CAD/CAM technique. J Prosthet Dent 2015; 114: 248-253.

11) Schlenz MA, Schmidt A, Rehmann A, Niem T, Wöstmann B. Microleakage of composite crowns luted on CAD/CAM-milled human molars: a new method for standardized in vitro tests. Clin Oral Investig 2019; 23: 511-517.

12) Russel WMS, Burch RL. The principles of humane experimental technique. London: Meuthen; 1959.

13) Strang G, Fix GJ. An analysis of the finite element method. J Appl Math Mech 1975; 55: 696-697.

14) Güth JF, Zuch T, Zwinge S, Engels J, Stimmelmayr M, Edelhoff D. Optical properties of manually and CAD/CAMfabricated polymers. Dent Mater J 2013; 32: 865-871.

15) Rosentritt M, Raab P, Hahnel S, Stockle M, Preis V. In-vitro performance of $\mathrm{CAD} / \mathrm{CAM}$-fabricated implant-supported temporary crowns. Clin Oral Investig 2017; 21: 2581-2587.

16) Wiegand A, Stucki L, Hoffmann R, Attin T, Stawarczyk B. Repairability of CAD/CAM high-density PMMA- and composite-based polymers. Clin Oral Investig 2015; 19: 2007 2013.

17) Quinn JB, Quinn GD. A practical and systematic review of Weibull statistics for reporting strengths of dental materials. Dent Mater 2010; 26: 135-147.

18) Amezua-Lasuen $X$, Iturrate-Mendieta $M$, Oriozabala-Brit JA, Garikano-Osinaga X, Martin-Amundarain I, SolaberrietaMendez E. Best-Fit Alignment in the Digital Dental Workflow. Advances in Design Engineering. Berlin: Springer; 2020.

19) Burns DR, Beck DA, Nelson SK, Committee on Research in Fixed Prosthodontics of the Academy of Fixed P. A review of selected dental literature on contemporary provisional fixed prosthodontic treatment: report of the Committee on Research in Fixed Prosthodontics of the Academy of Fixed Prosthodontics. J Prosthet Dent 2003; 90: 474-497.

20) Gough M. A review of temporary crowns and bridges. Dent Update 1994; 21: 203-207.

21) Beuer F, Schweiger J, Edelhoff D. Digital dentistry: an overview of recent developments for CAD/CAM generated restorations. Br Dent J 2008; 204: 505-511.

22) Li RW, Chow TW, Matinlinna JP. Ceramic dental biomaterials and CAD/CAM technology: State of the art. J Prosthodont Res 2014; 58: 208-216.

23) Basaran EG, Ayna E, Vallittu PK, Lassila LV. Load bearing capacity of fiber-reinforced and unreinforced composite resin CAD/CAM-fabricated fixed dental prostheses. J Prosthet Dent 2013; 109: 88-94.

24) Wimmer T, Ender A, Roos M, Stawarczyk B. Fracture load of milled polymeric fixed dental prostheses as a function of connector cross-sectional areas. J Prosthet Dent 2013; 110: 288-295. 
25) Bosch G, Ender A, Mehl A. A 3-dimensional accuracy analysis of chairside CAD/CAM milling processes. J Prosthet Dent 2014; 112: 1425-1431.

26) Edelhoff D, Schraml D, Eichberger M, Stawarczyk B. Comparison of fracture loads of CAD/CAM and conventionally fabricated temporary fixed dental prostheses after different aging regimens. Int J Comput Dent 2016; 19: 101-112.

27) Goryainova KE, Morokov ES, Retinskaja MV, Rusanov FS, Apresyan SV, Lebedenko IY. The micromorphological research of the internal structure of chairside CAD/CAM materials by the method of scanning impulse acoustic microscopy (SIAM). Open Dent J 2018; 12: 125-132.

28) Padma S, Umesh S, Asokan S, Srinivas T. Bite force measurement based on fiber Bragg grating sensor. J Biomed Opt 2017; 22: 1-6.

29) Umesh S, Padma S, Asokan S, Srinivas T. Fiber Bragg grating based bite force measurement. J Biomech 2016; 49: 2877-2881.

30) Podhorsky A, Rehmann P, Wostmann B. Tooth preparation for full-coverage restorations-a literature review. Clin Oral Investig 2015; 19: 959-968.

31) Stawarczyk B, Beuer F, Ender A, Roos M, Edelhoff D, Wimmer T. Influence of cementation and cement type on the fracture load testing methodology of anterior crowns made of different materials. Dent Mater J 2013; 32: 888-895.

32) Zhang Y, Mai Z, Barani A, Bush M, Lawn B. Fractureresistant monolithic dental crowns. Dent Mater 2016; 32
442-449.

33) Tsumita M, Kokubo Y, Vult von Steyern P, Fukushima S. Effect of framework shape on the fracture strength of implant-supported all-ceramic fixed partial dentures in the molar region. J Prosthodont 2008; 17: 274-285.

34) Jin XZ, Homaei E, Matinlinna JP, Tsoi JKH. A new concept and finite-element study on dental bond strength tests. Dent Mater 2016; 32: e238-e250.

35) Borba M, Duan Y, Griggs JA, Cesar PF, Della Bona A. Effect of ceramic infrastructure on the failure behavior and stress distribution of fixed partial dentures. Dent Mater 2015; 31: 413-422.

36) Abduo J, Bennani V, Waddell N, Lyons K, Swain M. Assessing the fit of implant fixed prostheses: a critical review. Int J Ora Maxillofac Implants 2010; 25: 506-515.

37) Schlenz MA, Schmidt A, Rehmann P, Wöstmann B. Fatigue damage of monolithic posterior computer aided designed/ computer aided manufactured crowns. J Prosthodont Res 2019; 63: 368-373.

38) Helkimo E, Carlsson GE, Helkimo M. Bite force and state of dentition. Acta Odontol Scand 1977; 35: 297-303.

39) Helkimo E, Ingervall B. Bite force and functional state of the masticatory system in young men. Swed Dent J 1978; 2: 167 175.

40) Manns A, Rojas V, Van Diest N, Rojas D, Sobral C. Comparative study of molar and incisor bite forces regarding deciduous, mixed, and definitive dentition. Cranio 2020: 1-8. 